\title{
SLOWENISCHE ORTSNAMEN IN LATEIN ${ }^{1}$
}

\section{Einleitung}

Da Latein noch immer auch eine Kommunikationssprache (in geschriebener als auch gesprochener Form) ist, muß sie weiterentwickelt werden, um auch die Erscheinungen der modernen Zeit ausdrücken zu können. Einer der Bereiche, der normiert werden muß, ist der Bereich der Eigennamen.

Weltweit erfaßt die gegenwärtigen erdkundlichen Namen Eggers Lexicon nominum locorum ${ }^{2}$. Weil dessen Umfang begrenzt ist, sind die slowenischen erdkundlichen Namen nicht gerade in großer Zahl vertreten. Das Ziel dieser Arbeit ist eine Erweiterung zum obengenannten Lexikon zu schaffen, die den slowenischen Raum erfassen sollte. Ich habe mich auf die Ortsnamen innerhalb des slowenischen Staatsgebietes begrenzt, d.h. nicht vollständig den slowenischen nationalen Raum bearbeitet.

\section{Die Lateinisierung der heutigen slowenischen erdkundlichen Namen}

Erdkundliche Namen ohne überlieferte lateinische Namen werden auf eine der zwei folgenden Weisen lateinisiert:

1) Namen mit lexikaler Bedeutung (diese Namen dienen zur Identifikation, beschreiben außerdem aber noch ein Merkmal des Ortes) können ins Lateinische übersetzt werden, wenn in Latein eine gute Entsprechung gefunden werden kann. Wortgruppen können gänzlich übersetzt werden, wenn möglich (wenn alle einzelnen Wörter Gattungsnamen sind), andertfalls werden nur die Bestandteile, die Gattungsnamen sind, übersetzt.

1 Der Artikel ist eine Zusammenfaßung des zweiten Teiles meiner Diplomarbeit (Filozofska fakulteta, Ljubljana, Oddelek za klasično filologijo, bei akad. Prof. Dr. Kajetan Gantar), in dem die erdkundlichen Namen behandelt sind, die nicht aus der Antike überliefert wurden, noch keine lateinische Form haben und daher neu gebildet werden müssen.

2 C. Egger, Lexicon nominum locorum, Libraria Vaticana 1977. 
2) Wenn der Name nicht übersetzbar ist, wird er (phonologisch und morphologisch) der lateinischen Sprache angepaßt: Lautsymbole, die in Latein nicht vorhanden sind, entfallen oder werden ausgetauscht, Präfixe bzw. Suffixe werden hinzugefügt, wenn es sich um kein Simplex handelt, oder der Name wird zu den Wörten klassifiziert, die die Kasus nicht mit lautlichen Endungen ausdrücken.

\subsection{Das Übersetzen von Namen ins Lateinische}

Viele Orte, Gewässer und Berge wurden nach einem ihrer Merkmale benannt. Wahrscheinlich trugen alle Namen einst eine Bedeutung, nur kann sich die Bedeutung mit der Zeit verwischen; viele Namen sind überdies auch aus fremden Sprachen übernommen worden. Bei den Namen, die nicht nur zur Identifikation dienen, aber auch der Charakterisierung, ist der anderssprachige Leser benachteiligt (obwohl die lexikale Bedeutung heutzutage wegen Veränderungen schon ihre Aktualität verloren haben könnte). Aus diesem Grund sollte man diese Namen übersetzen, soweit eine lateinische Entsprechung gefunden werden kann.

Am wenigsten Schwierigkeiten gibt es bei der Übersetzung von Simplexen, die ursprünglich Gattungsnamen waren: Reka - lat. Flumen -inis; Dolina - Vallis -is; Blato Lutum -i; Vrata - Porta -ae; Breg - Clivus -i; Breza - Betul(l)a -ae; Ceste - Viae arum; $\breve{C}_{e l o}{ }^{3}$ - Frons -tis. Nicht für alle Namen kann ein entsprechender einwortiger, lateinischer Ausdruck gefunden werden. Ein Bespiel dafür ist die häufige Hydronymie bzw. der Ortsname Bistra und dessen Diminutivum Bistrica. Das Adjektiv bister umfaßt die Bedeutung zweier Adjektive 'schnell, fließend', aber auch 'klar, sauber'. Auf ähnliche Übersetzungschwierigkeiten stößt man auch bei den Namen Draga, Dobrava, Gmajna. In einigen Fällen ist in Latein nur ein lateinischer Ausdruck vorhanden, wo das Slowenische über zwei oder mehr Wörter verfügt, die eine ähnliche Bedeutung haben, aber doch einen kleinen Unterschied aufweisen. Das ist natürlich ein übliches Übersetzungsproblem, verständlich zumal die Sprachen auch verschiedenen Zeiten angehören. Solche Fälle müssen einzeln behandelt werden. In historischen Schriften ist der slowenische Name Brda mit Colles überstezt worden, die gleiche Übersetzung wurde für Gorice gebraucht. Die Wörterbuchbedeutung von Brda und Gorice ist zwar ähnlich ('hügelreiche Landschaft'), doch tragen beide eine zusätzliche Bedeutungskomponente: gorice 'eine hügelreiche Landschaft, meist mit Weingärten (lat. collis vinearius); brdo 'ein unhoher, oft länglicher Hang'. Nun gibt es aber noch die Ausdrücke hrib und grič, die gleichfalls mit dem lateinischen collis übersetzt werden können. Es bleibt noch der Ausdruck clivus, der den slowenischen Ausdrücken breg und klanec 'geneigte Landschaft, Hang' entspricht. bezeichnet einen steilen Hang, Teil eines Berges oder eine Bergwand; deshalb ist er oft als Benennung eines Berges zu finden. 
Bei den mehrwortigen Ortsnamen kann nur ein Wort ein Gattungsnamen sein. Dazu einige Beispiele:

mesto > urbs (größere Stadt), oppidum (kleinere Stadt), -polis (in der Antike für Städte, die nach einer Person oder einem Gott benannt wurden, aber auch sonst, besonders wenn sie von griechischen Namen abgeleitet wurden: Philippopolis, Nicopolis, Megalopolis. Heutzutage für Städte, die von Personennamen abgeleitet wurden: San Francisco - Franciscopolis, Bismarck - Bismarkopolis, und auch für viele andere: Buenos Aires - Bonaeropolis, Cape Town - Promuntoriopolis, Feuchtwangen - Hydropolis. Für slowenische Ortsnamen findet diese Benennung nur wenig Anwendung, da Orte, deren Namen von Eigennamen abgeleitet wurden, eher die größe von Dörfern als von Städten haben. Von den größeren Städten kommt in Betracht nur Kidričevo Kidritzopolis, Hrastnik - Quercopolis, während für einige älteren Städte diese Benennung schon überliefert wurde: Locopolis - Škofja Loka, Lithopolis - Kamnik);

vas > vicus, pagus (der Ausdruck pagus hat noch eine breitere Bedeutung: mehrere Dörfer, Bezirk, in der Schweiz Kanton ("omnis Civitas Helvetia in 4 pagos divisa est"). Der Ausdruck ist eher ein Terminus zur Bezeichnung von administrativen Teilen, wie im Slowenischen z.B. občina, und nicht geeignet für Eigennamen);

trg > forum, mercatum (geeigneter ist der Ausdruck forum, der schon in antiken Eigennamen in Italien und in den Provinzen häufig vorkam: Forum Appii, Forum Aurelium, Forum Gallorum, Forum Iulii);

gora, gorovje > mons, montes, Alpes (das Substantiv Alpe ist zwar im Slowenischen noch immer ein Eigennamen, wird jedoch schon fast als Gattungsnamen verstanden 'hohe Berge', deshalb ist es auch zu Doppelbenennungen gekommen, z.B. Kamniške planine = Kamniške Alpe);

planina > mons, montes; pascua (beide Bedeutungen des Terminus treten in slowenischen geografischen Namen auf; als (meist im Plural) 'hohe Berge' - übersetzt als montes; und in der Bedeutung 'Bergweide' als pascua, z.B. Velika planina - Pascua Magna);

grič > colliculus;

hrib > collis;

breg, klanec > clivus;

sedlo, sleme > iugum;

dolina, dol > vallis (die Wörter dolina und dol treten auch als Einworteigennamen auf; dol hat zwar die gleiche Bedeutung als dolina, ist im Wörterbuch jedoch als veraltet markiert. Da der Name nicht mehr zum neutralen Wortschatz gehört, sollte man ihn besser unübersetzt lassen, wenn er als Einworteigennamen auftritt);

kotlina > convallis;

jama > caverna, specus, spelunca (in mittelalterlichen Texten wurde dieses Wort mit antrum übersetzt, ein Terminus, der im klassischen Lateinischen nur in poetischen Texten verwendet wird); 
grad > castrum;

most > pons;

krajina, pokrajina > regio;

otok > insula;

$k o t>$ angulus;

gozd > silva, saltus (saltus bedeutet 'Bergwald', 'waldreiches Gebirge', "regio montana et silvosa");

\section{$\log >$ nemus;}

jezero > lacus;

vir, izvir (vir ist ein weniger gebrauchtes Synonym von izvir) > fontes;

studenec (fast synonym mit dem obengenannten vir, izvir 'Wasser, daß aus der Erde an die Oberflache fließt/quillt', ein studenec ist üblicherweise ein kleiner izvir) > fons;

reka > amnis (ein größerer Fluß oder schnell fließendes Gewässer), flumen, fluvius;

potok $>$ rivus;

toplice > aquae;

beli, črni > albus, niger;

mali, veliki > parvus, magnus; minor, maior (der Komparativ wird verwendet, wenn betont werden soll, daß das Objekt aus zwei Teilen besteht, für Ortsnamen also, die in Paaren auftreten, z.B. Italia Superior, Italia Inferior, Longaticum Superius, Longaticum Inferius).

stari, novi > antiquus, novus;

zgornji, spodnji ( gor(e)nji, dol(e)nji) > superior, inferior;

mrzli, topli > frigidus, calidus;

goli > nudus;

suhi $>$ aridus;

ravni $>$ aequus, planus;

dolgi > longus;

Mehrwortige Namen, deren Teile ursprünglich Gattungsnamen waren, kann man gänzlich übersetzen: Suha krajina > Regio Alba; Novi trg > Forum Novum; C̆rni vrh > Vertex Niger; Travna Gora > Mons Gramineus.

Slowenische Ortsnamen scheinen in der Regel von Gattungsnamen abgeleitet $\mathrm{zu}$ sein: Jezer-sko, Kamn-ik, Gol-ica. Aus der gleichen Wurzel konnten durch Hinzufügung von Suffixen mehrere Ableitungen geformt werden: Brezje, Brezovica, Breznik. Beim Übersetzen solcher Namen sollte versucht werden, die entsprechenden lateinischen Suffixe $\mathrm{zu}$ finden, wobei sehr auf stilistische und regionale Besonderheiten geachtet wer- 
den sollte. Ein allzu großer Übersetzungseifer könnte zu Schwierigkeiten bei der Identifikation der Orte führen, was doch der Hauptzweck der Benennung war. Deshalb ist es bei Ableitungen ratsam, nicht unbedingt zu übersetzen, aber in Handbüchern nur ihre Bedeutung erläutern bzw. die Basis übersetzen ${ }^{4}$ (außer in einigen Fällen, in denen eine Verwechslung mit ähnlichen Namen ausgeschlossen ist).

Übersetzt können einige Arten von Ableitungen werden, vor allem Namen, die durch Konversion aus Adjektiven entstanden sind (Belo, Golo, Visoko,...). Gewöhnlich sind sie Neutra, aber auch Feminina (Črna, Bela, Ravne). Auf diese Weise sind auch einige alten römischen Namen entstanden: Albula, Alsium.

Ravne > Aequae -arum (Planum -i wäre die Übersetzung für Plano, entstanden aus dem Adjektiv plan, das ein nahes Synonym von raven ist); Visoko $>$ Altum -i, das Gegenteil Globoko $>$ Profundum -i; Golo $>$ Nudum -i; Bela $>$ Alba -ae; Črna $>$ Nigra -ae.

Weiter gibt es auch einige aus substantivischer Basis konvertierte Adjektive: Kačje $>$ Colubrinum -i (der antike Name der Insel Culubrinum - $i=$ Kačji otok/Schlangeninsel entstand aus dem Grundwort coluber); Blatno $>$ Luteum -i; Kozje $>$ Capreum -i. ${ }^{5}$

Genauso ist es möglich Diminutiva zu übersetzen, wenn das Grundwort übersetzt wurde: $\operatorname{Trg}>$ Forum -i, daraus Tržič $>$ Forulum -i; Glava $>$ Caput -itis, Glavica $>$ Capitulum -i; Lipa $>$ Tilia -ae, Lipica $>$ Tiliola -ae.

In anderen geographischen Namen werden Übersetzungen noch am häufigsten bei Namen von Bergen verwendet, bei denen die lexikale Bedeutung noch deutlich faßbar ist. Der Name wird als Adjektiv mit dem Bestimmungswort Mons übersetzt: Snežnik > Mons Nivosus.

Zusammensetzungen sind im Slowenischen ein unproduktives Wortbildungsmuster, es gibt jedoch einige solche, die übersetzt werden können: Portorož > Portus Rosae (der Genitiv rosae als kollektiver Singular) oder Portus Roseus ${ }^{6}$; Triglav > Mons Triceps; Mokronog - in historischen Schriften oft in der deutschen Übersetzung Nassenfuß gefunden. Diese Art von ursprünglich adjektivischen Zusammensetzungen gibt es in Latein nicht, anselle dessen gibt es den Ablativus qualitatis. Da dieses Wortbildungsmuster in Latein nicht üblich war, habe ich den Namen unübersetzt gelassen und ihn der Klasse der Indeklinabilia zugeordnet: Mocronog, $n$.

Präpositionalbildungen waren schon in der Antike beliebt, und zwar mit den Suffixen -ius und -ium (Plural. -ia): submoenium, intermundia; so auch bei Ortsnamen: Interamnium, Subsilvania.

Z.B. Graz (Gradec) - Gradecium -ii, n /.../ (grad = locus circumsaeptus, circummunitus; gradec = forma deminuta: castellum (Egger, Lexicon, 134).

5 Namen von dieser Basis gab es schon in der Antike sehr viele: Capreae (Capri), Caprae (Koper), ...

6 Ähnlich wie z.B. Port-Louis - Portus Ludovici, vel Portus Ludovicianus (Egger, Lexicon, 245). 
Medvode > Interaquae -arum oder Interamn(i)a -ae ${ }^{7}$. Ähnlich ist es mit Medvodje, das mit Interamnium -ii übersetzt werden kann und somit ein Neutrum bleibt. Weil es im slowenischen Raum keinen Ort mit dem Namen Medrečje gibt, kann als Basis amnis anstelle von aqua verwendet werden.

Podbrdo > subcollium -ii; Preddvor > Praecuria -ae; Zagorje > Transmontium -i; Podkoren > Subradicium -ii; Zagradec > Postgradecium -ii (bei Zusammensetzungen kann auch nur ein Teil der Basis übersetzt werden ${ }^{8}$, weil der Name Gradec nicht übersetzt wird). Auch gibt es Bildungen aus Präpositionen und Gattungsnamen: Prekmurje, Zasavje. Aus alten Schriften ist ersichtlich, das diese anders übersetzt wurden: durch zweiwortige Namen, ohne Praposition: Čezsoča > Ultra Isontium, Posavje (das slowenische Präfix po- signalisiert, daß es sich um das Gebiet entlang dem Fluß handelt) > Savia -ae. Nach der Analogie dann auch Dravia -ae für Podravje und Muria -ae für Pomurje.

Präpositionale Namen: Auch die Übersetzung sollte die Präposition enthalten: $\mathrm{Na}$ Hribu > In Colle; Na Dravi > In Dravo; Pod Gozdom > Sub Silva; Pri Jezeru > Ad Lacum; $V$ Dolin $i>$ In Valle. Doch steht fast obligatorisch vor dem Namen die Artsbestimmung (meist vicus): "hic est vicus In Colle" (so auch im Slowenischen: "to je vas Na Hribu").

\subsection{Die Angleichung der Namen an die lateinische Sprache}

Angeglichen werden Namen, bei denen die Verbindung zum Urwort (ursprünglichen Gattungsnamen) nicht mehr durchblickt (wegen sprachlicher Veränderungen oder, weil sie aus einer fremden Sprache übernommen worden sind), und einige Bildungen aus Gattungsnamen; besonders typisch für den slowenischen Raum sind Ableitungen.

\subsubsection{Angleichungen in der Orthographie}

Für die Angleichung in der Orthographie gelten einige allgemeine Regeln ${ }^{9}$; ich führe dazu auch einige Regeln an, die für die slowenische Sprache gelten:

- $\check{s}$ : wird s geschrieben nach dem Beispiel der alten Römer und Griechen, z.B.: Yerushalaim - Ierusalem): Šoštanj $>$ Sostanium -ii, Menge ̌̌ $>$ Mengesum -î.

- $\check{c}$ : wird tz geschrieben (z.B. Tzechoslovakia): Črnomelj > Tzernomelium -ii, Čemšenik > Tzemsenicum -i, Bača > Batza.

7 Orte mit Namen wie Interamna, Interamnium, Interamnia, gab es in der Antike mehrere, z.B. das heutige Teramo, Terni.

8 Z.B. Hohenstadt - Altostadium -ii (Egger, Lexicon, 145).

9 Egger, Lexicon, 8-9. 
- $k$ : wird c geschrieben: Komna > Comna -ae, Kropa > Cropa -ae, Loka > Loca -ae; vor den Lauten $e$ und $i$ bleibt wegen der verschiedenen Lautbildung in den modernen Sprachen k (z.B. Kiel - Kilonia -ae, Bangkok - Bancokium -ii): Brkini > Berkines -ium. Wenn $\mathbf{k}$ am Wortende steht (vor dem Suffix), füge ich das lateinische Suffix -ium (-ius) hinzu: Rakek > Rakekium -ii, Bertoki > Bertokii -iorum. Jedoch sind bei Namen, in denen das $\mathbf{k}$ Teil des Suffixes ist (im Slowenischen häufig Ableitungen auf -ik), die lateinischen Suffixe -icum und -icus ${ }^{10}$ treffender.

- z: bleibt z: Polzela > Polzela -ae, Trzin > Terzinum -i, Razor > Razor -oris.

- c: diesen Laut gab es im klassischen Latein nicht; das Symbol wurde k gesprochen. Dieser Laut wurde gegen Ende der Antike vor Vorderzungenvokalen zu einem weichem c. Unsere Vorfahren übernahmen die Namen in der neuen Form (z.B. der Name Celeia - slow. Celje); diese Aussprache erhielt sich. Wenn im slowenischen Name das $c$ nicht vor $e$ oder $i$ steht, werden diese Laute dem $c$ beigefügt: Gradec $>$ Gradecium -ii, Lisca > Liscia -ae; vor $i$ und $e$ bleibt das $c$ : Lucija > Lucia; auch die Lautgruppe $-t i$ - wird vor einem Vokal zu einem weicheren - $c i$ - . Die Schreibweise mit dem Buchstaben - $t$ - findet Verwendung beim lateinischen Suffix -itia in der Angleichung einiger slowenischer Namen auf -ica: Gorica $>$ Goritia -ae, Planica > Planitia -ae.

- ž: wird z geschrieben: Sežana > Sezana -ae, Mežica > Mezicia -ae, Domžale > Domzalae -arum. $\check{Z}$ ist die stimmhafte Variante des stimmlosen Sonanten $\check{s}$, der $s$ geschrieben wird. Diese Schreibweise ist auch begründet durch die Analogie zur Schreibweise von $d z ̌$ als $d z$, die sich schon vollens durchgesetzt hat. In übernommenen Wörtern ist das $\check{z}$ aus $s$ entstanden: rosa > roža, Risano > Rižana.

- Ein Halblaut wird mit $e$ widergegeben. Der im Slowenischen unbeständige Halblaut bleibt im Latein erhalten: Gradec $>$ Gradecium -ii, Črnomelj > Tzernomelium -i; er wird auch geschrieben vor jedem $r$, das nicht vor einem Vokal steht, auch wo es im Slowenischen nicht geschrieben wird (in der Wortmitte): Trbovlje > Terboulia -ae.

- $v$ : da sich ab dem Humanismus die Unterscheidung zwischen $u$ und $v$ durchgesetzt hat, das $v$ zwischen Vokalen, andertfalls aber $u$ zu schreiben, halten wir uns auch hier an diesen Grundsatz: Die Orthographie mit $v$ vor Vokalen: Ledava > Ledava -ae, Olševa > Olseva -ae, und $u$ vor Konsonanten (überall, wo im Slowenischen ein Diphtong oder zweilippiges $u$ gesprochen wird): Sevnica $>$ Seunicia -ae, Pivka $>$ Piuca -ae.

- lj, nj: wird li, ni geschrieben: Ljutomer > Liutomerum -i; in der Zusammensetzung -lji-, -nji- fallt ein $i$ ab: Radovljica > Radoulicia -ae; am Wortende entfällt ein $i$ als Teil des Suffixes -ium: Ljubelj > Liubelium -ii. 


\subsubsection{Morphologische Angleichung}

Das Genus: das grammatische Genus kann sich ändern; hierzu gibt es keine festen Regeln. Gewöhnlich sind im Latein die Namen von größeren Flüssen Maskulina, von kleineren Flüssen und Bächen Feminina. Die antiken Namen der größten slowenischen Flüsse waren Maskulina: Dravus -i, Savus -i, kleinere Flüsse aber Feminina: Corca -ae. Im Slowenischen sind die Namen für Flüsse konsequent Feminina: Drava, Sava. Lateinische Ortsnamen sind meist Feminina oder Neutra, jedoch ist das keine feste Regel.

Deklination: Einige Suffixe bleiben unverändert, z.B. die meisten Feminina mit der Endung -a behalten die Endung und das Genus und werden nach der a-Deklination dekliniert; sie werden gegebenfalls nur orthographisch angeglichen: Mirna > Mirna -ae, Sora $>$ Sora -ae, Metlika $>$ Metlica -ae, Divača $>$ Divatza -ae.

Anderen Namen, vor allem Maskulina, die im Nominativ eine nicht stimmhafte Endung haben, wird eine Endung angehängt, und zwar an Ortsnamen meist - um, an Namen für Berge meist -us: Kobarid $>$ Cobaridum $-\mathbf{i}^{11}$, Tolmin $>$ Tolminum $-\mathbf{i}$, Ankaran $>$ Ancaranum -i, Mangart > Mangartus -i; die Verbindungen $-n j$ und $-l j$ am Wortende ergeben die Endung -ium, -ius: Tuhinj > Tuhinium -ii; Namen mit $-r$ im Auslaut (vor allem, wenn die Kasus außer Nominativ mit $-j$ - gebildet werden), werden nach der dritten (gemischten) Deklination dekliniert: Razor > Razor -oris.

Noch einige der häufigeren Prä- und Suffixe:

-ica: das slowenische Suffix -ica hat verschiedene Bedeutungen, z.B. eine diminutive (Reka - Rečica), es substantiviert das Adjektiv und bezeichnet den Träger einer Eigenschaft (Škrlatica) u.ä. Diminutiva können als solche übersetzt werden: Vodice > Aquulae -arum, Rečica $>$ Amniculus -i, oder das Suffix wird lateinisiert alls -icia oder -itia. Für Ableitungen mit adjektivischen Stämmen ist in den meisten Fällen das lateinische Suffix -itia (mit der Endungsvariante -ities -ei) das geeignete und im Plural -itiae, das im Latein an adjektivische Basen gehängt wurde ${ }^{12}$ und eine ähnliche Bedeutung hat, z.B. das Substantiv planities abgeleitet vom Adjektiv planus (plan-us). Im Slowenischen gibt es die gleiche Wurzel (plan -a -o) 'Ebene', daher würde dem slowenischen Name Planica, das lateinische Planitia entsprechen. Mit dem Suffix -icia wurden substantivische Ableitungen gebildet ${ }^{13}$. Im Slowenischen geht es um eine Ersetzungsableitung, die ein Adjektiv ersetzt (Konjski hrib > Konjščica), oder als direkte Ableitung von einem Substantiv (korito > Koritnica), auch aus Eigennamen (Radov-(lj)ica). Weil es jedoch bei einigen Wörtern schwer festzustellen ist, aus welcher

11 Der Name stammt angeblich aus lat. capretum, heutig furl. Chavored (Ramovš, Kratka zgodovina, 39).

12 bland-itia, dur-ities.

13 Ursprünglich zur Bildung von Adjektiven aus substantivischen Basen, dieses Suffix dient auch zur Bildung von Ortsnamen aus Personennamen: Vandalicia < Vandali. 
Basis sie entstanden sind, ist es besser einheitlich das Suffix -icia zu verwenden (außer in Fällen, in denen sich das Suffix -itia schon durchgesetzt hat: Goritia, Planitia). In dieser Weise bleibt die lateinische Form näher der slowenischen: Selnica > Selnicia -ae, Rodica > Rodicia -ae, Bistrica - Bistricia ${ }^{14}$-ae u.ä.

Ableitungen, die Berge benennen, können in Latein mit einer zweiwortigen Benennung widergegeben werden: $\breve{S} k r l a t i c a>$ Purpureus mons ${ }^{15}$.

$-i j a>-i a$ (wenn das slowenische $i$ betont ist, ist das lateinische $i$ lang): Litija $>$ Litia -ae, Lucija > Lucia -ae, Idrija > Idria -ae.

-nja > -nia, z.B. Mislinja > Mislinia -ae, daher auch Savinja > Savinia -ae, Dravinja > Dravinia -ae, die waren ursprünglich Adjektive zu Sava, Drava: Savina, Dravina (Fluß).

-je > -ia: Trebnje > Trebnia -ae, Velenje > Velenia -ae, Begunje > Begunia -ae, Mozirje > Moziria -ae, Posavje > Savia -ae; -je ist ein Suffix mit possessiver Bedeutung, gleich den lateinischen -ius -ia -ium; bei einigen Namen ist das - $e$ die Pluralendung der Feminina (Radlje), doch sind in der Geschichte diese zwei Formen zusammengefallen und haben sich vermischt, deshalb können beide in gleicher Art lateinisiert werden.

$-o>-u m:$ Senovo $>$ Senovum -i, Cerkno $>$ Cercnum -i, Trebno $>$ Trebnum -i.

-sko > -scum: Wenn der Stamm auf einen Konsonanten auslautet, wird ein $i$ eingeschoben, das die schwer aussprechbare Konsonatengruppe vereinfacht: Pragersko $>$ Prageriscum -i, Ravensko $>$ Raveniscum -i, Sotelsko $>$ Soteliscum -i. ${ }^{16}$

$-i k>-i c u m$ : Vojnik > Voinicum -i, Javornik > Iavornicum -i, Golnik > Golnicum $-\mathbf{i}$.

$-e c>-e c i u m$ : Nach dem Muster Gradecium -ii für Gradec können noch andere Namen gebildet werden: Bovec $>$ Bovecium -ii, Rogatec $>$ Rogatecium -ii, Žalec $>$ Zalecium -ii.

$-c i>-i c i i$ Im Nordosten Sloweniens (in der Umgebung von Murska Sobota und Ptuj) gibt es viele Namen, die von Eigennamen durch das Suffix - ci abgeleitet sind (auch -ovci und von weiblichen Basen mit dem Suffix -inci); In alten Schriften kann der lateinisierte Name umschrieben gefunden werden: Ivanovci - Villa Johannis ${ }^{17}$, doch

14 Beim Anon. Rav. (IV c. 19, p. 115-6) gibt es einen antiken Namen Bustricius, der nach Meinung von Wissenschaftlern nicht slowenisch mit behaltenem -u- ist (Bezlaj, Slovenska vodna imena I), obwohl das phonetisch möglich wäre, denn ursprünglich hatte der Name einen Vokal -y- (Bystrica), der ungefähr zwischen $i$ und $u$ artikuliert wird. Vor langer Zeit hat sich das y durch u substituiert und wurde dann gewöhnlich mit u geschrieben, im Slowenischen hat sich das y zu einem i entwickelt.

15 Nach einer Theorie stammt auch der Name Kočna aus lat. coccinus 'škrlaten/scharlachfarben' (Pohl, Slavische und Romanische Oronyme in den Karawanken; 178). Die Theorie fundiert auf der Tatsache, daß dieses Wort in germanischen Sprachen häufig in Benennungen von Bergen vorkommt.

Ähnlich wie z.B. Minsk - Miniscum, Tomsk - Tomiscum (Egger, Lexicon, 203, 313). 
geht dabei die plurale Bedeutung verloren. Das lateinische Adjektivsuffix -icius trägt unter anderem auch die Bedeutungskomponente der Angehörigkeit, Ursprungs und ist in diesem Fall geeignet (im Plural): Adrijanci $>$ Adrianicii -orum, Andrejci $>$ Andreicii -orum, Petrovci $>$ Petrovicii -orum, Domajinci $>$ Domainicii -orum.

Die gleichen Regeln gelten auch für mehrwortige Namen. Oft enthalten sie von Eigennamen abgeleitete Adjektive. Einige Ortsnamen haben schon eine überlieferte Adjektivform: ljubljanski - Labacensis -e, dravski - Dravensis -e, ptujski - Poetoviensis -e; in neuen Verbindungen, z.B.: Ljubljansko barje > Palus Labacensis, Dravska dolina > Vallis Dravensis, Ptujsko polje > Campus Poetoviensis. Diese Formen können als Muster für neue Bildungen genommen werden: Savensis (aus Savus, in Analogie zu Dravensis): Savska dolina > Vallis Savensis, Savski vrh > Vertex Savensis.

Noch einige mehrwortige Namen, die neu aus überlieferten Wurzeln gebildet werden:

Škofljica: aus dem Wort škof - lat. episcopus ${ }^{18}$. Nach dem Beispiel Episcop-lie für Škof-lje (mit Hinsicht auf die Endung ist besser Episcoplia -ae), wäre Škof-ljica >

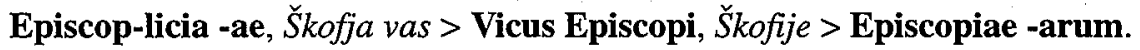

Pomurje, Podravje, Posavje: Muria -ae, Dravia -ae nach der Analogie zu Posavje $>$ Savia -ae. Prekmurje > Ultra Murium (ähnlich wie Čezsoča > Ultra Isontium), aber auch zusammen geschrieben Ultramurium -ii.

Murska Sobota, Mursko polje: Sobota Muriensis, Campus Muriensis.

Dravograd: Dravogradum $-\mathbf{i}^{19}$.

Slovenj Gradec (das - $j$ in Slovenj ist ein Adjektivsuffix, das dem lateinischem Suffix -ium entsprechen könnte): Slovenium Gradecium, doch ist in Latein die Zusammensetzung Slovenogradecium -ii usueller ${ }^{20}$.

Einige gleichnamigen Ortsnamen enthalten eine Bestimmung, die signalisiert, in der Nähe welchen größeren Ortes der Ort liegt: Bistrica ob Sotli > Bistricia ad Sotlam. Wenn jedoch die Bestimmung eine Region ist, wird gewöhnlich der Genitiv verwendet: Črna na Koroškem > Nigra Carinthiae ${ }^{21}$.

An vielen slowenischen Flüssen liegen gleichnamige Orte (Soča, Sava, Krka). In solchen Fällen bleibt die Endung - $a$ erhalten, auch wenn der lateinische Name des

Zelko, Histor. topografija Slov. I.

18 Von dieser Wurzel abgeleitete Namen sind geläufig auch in anderen Sprachen, z.B. Bischofshofen Episcopi Curia (Egger, Lexicon, 54).

19 Wie Oberdrauburg - Dravoburgum -i; Volgograd - Volgogradum -i (Egger, Lexicon, 224, 332).

20 Ähnlich wie z.B. Hradec Kralove - Reginogradecium -ii (Egger, Lexicon, 147).

21 Wie z.B.: Alexandrette > Alexandria ad Issum; Augusta > Augusta Georgiae (Egger, Lexicon, 23, 40). 
Flusses ein Maskulinum ist: Sava > Sava -ae (vicus), Savus -i (fluvius); Soč $a>$ (Ae)sontia -ae (vicus), (Ae)sontius -ii (fluvius) ${ }^{22}$;

Ethymologische Untersuchungen haben verschiedene Theorien über den Ursprung einzelner urslawischer Namen aufgestellt; darunter scheinen einige mehr, andere weniger glaubwürdig:

Sotla ist vermutlich aus dem urslawischen *Sontula entstanden; Flußnamen mit dem Suffix - ula sind keine Besonderheit im antiken Europa: Vistula - Visla. Das Suffix -l- war nicht immer ein Diminutionssuffix, es drückt auch Zugehörigkeit, Ähnlichkeit u.a. aus, also ähnlich wie im Slowenischen das Suffix -ica.

Der Name Horjul wurde auf das antike Forum Iulii zurückgeführt, einige sahen eine Verbindung zu Ariolo, phonologisch käme auch das deutsche Heerlen in Betracht, in der Antike Coriovallum. Doch sind das nur Vermutungen, keine Rekonstruktion ist endgültig ${ }^{23}$.

Mit großer Sicherheit stammt dagegen Šempas aus Sanctus Bassus (šent- aus sanctus hat in einigen Verbindungen das -t verloren; gleich *Šent-Mihael > Šmihel u.ä.).

Trotzdem ist es bei den meisten dieser Namen ratsam, sie aufgrund ihrer gegenwärtigen Form zu lateinisieren, andertfalls entfernt sich die lateinische Übersetzung zu weit von der heutigen slowenischen Form, jedoch auch weil ethymologische Rekonstruktionen oft nicht endgültig sind, aber nur Zwischenlösungen oder Vermutungen formulieren, die durch neue Forschungen bestätigt oder widerlegt werden können.

\section{Bibliographie}

Antibarbarus der Lateinischen Sprache. Basel 1905.

Atlas of the Classical World (ed. A. A. M. van der Heyden, H. H. Schullard). Nelson 1960.

Atlas Slovenije. Ljubljana 1985.

Bezlaj, F.: Slovenska vodna imena I, II. Ljubljana 1956.

Blaznik, P.: Historična topografija slovenske Štajerske in jugoslovanskega dela Koroške do leta 1500 I, II, III. Maribor 1986.

Du Cange, C.: Glossarium mediae et infimae Latinitatis I-X. Graz 1954 (Unveraenderter Nachdruck der Ausgabe von 1883-1887).

Egger, C.: Lexicon nominum Locorum. Officina libraria Vaticana 1977.

22 Genauso werden andere gegenwärtige Orte benannt, die an gleichnamigen Flüssen liegen: Hudson > Hudsonia (urbs), Hudsonius (fluvius); Illinois > Ilinoesia (civitas), Ilinoesius (fluvius).

23 Bezlaj, Slov. vodna imena I. 
Gantar, K.: Nekaj misli o pisavi antičnih imen in strokovnih izrazov. Arheološki vestnik 30 (1979), 584-558.

Kos, M.: Gradivo za historično topografijo Slovenije (Za Kranjsko do leta 1500) I, II, III. Ljubljana 1975.

Leumann-Hoffmann-Szantyr: Lateinische Grammatik I, II. Muenchen 1965.

Lubas, W.: Svojilna pripona -ica v južnoslovanski toponomastiki. Jezik in slovstvo 11 (1966), 173-176.

- - : O izmenični izpeljavi in konverziji v slovenskih krajevnih imenih. Jezik in slovstvo 12 (1967), 108-110.

- - : Očetnoimenska krajevna imena z obrazili -ci, -ovci, -inci v slovenskem jeziku. Jezik in slovstvo 14 (1969), 72-74.

Ludvik, D.: Nekaj krajevnih in vodnih imen. Slavistična revija 9 (1956), 189-195.

Normae ortographicae et ortotypicae Latinae. Vox Latina 27 (1991), 2-13.

Pohl, H. D.: Slavische und Romanische Oronyme in den Karawanken (und umliegenden Gebirgsgruppen). Slavistična revija 33 (1985), 178-184.

Ramovš, F.: Historična gramatika slovenskega jezika II. Konzonantizem. Ljubljana 1924.

- - : Kratka zgodovina slovenskega jezika I. Ljubljana 1936.

Thesauri linguae Latinae supplementum: Nomina Propria Latina (C-Cyzistra). Lipsiae 1909-1931.

\author{
Povzetek
}

LATINSKA IMENA SLOVENSKIH KRAJEV

Ker je latinščina še vedno jezik komunikacije, je treba normirati tudi zapisovanje današnjih lastnih imen. Današnja zemljepisna lastna imena so zajeta $\mathrm{v}$ leksikonu C. Eggerja (Lexicon nominum locorum). Zaradi omejenega obsega leksikona in majhnosti slovenskega prostora so slovenska krajevna imena zastopana zelo skromno. V prispevku, ki je povzetek dela diplomske naloge, navajam nekaj pravil za latinjenje današnjih zemljepisnih lastnih imen na državnem ozemlju Slovenije, in sicer tistih, ki še nimajo izpričane latinske oblike. Imena, ki imajo v slovenščini jasen leksikalni pomen (npr. Reka, Dolina) prevedemo, če obstaja latinski izraz z vsaj približno enakim pomenom. Prevesti je mogoče tudi imena, ki so nastala kot zloženke iz občnih imen (Triglav), imena, tvorjena iz predložne zveze (Podkoren), ter nekatere izpeljanke iz občnih imen (Visoko, Kozje). Druga imena prilagodimo zakonitostim latinskega jezika $v$ pisavi in oblikoslovju. Navajam pravila za zapisovanje posameznih slovenskih jezikovnih znakov, ki jih v latinščini ni, ter pravila latinjenja nekaterih pripon in končnic. 